\title{
Parkin cooperates with GDNF/RET signaling to prevent dopaminergic neuron degeneration
}

\author{
Durga Praveen Meka, ${ }^{1}$ Anne Kathrin Müller-Rischart, ${ }^{2}$ Prakash Nidadavolu, ${ }^{1}$ Behnam Mohammadi, ${ }^{1}$ Elisa Motori, ${ }^{3}$ \\ Srinivas Kumar Ponna, ${ }^{1}$ Helia Aboutalebi, ${ }^{1}$ Mahmoud Bassal, ${ }^{1}$ Anil Annamneedi, ${ }^{1}$ Barbara Finckh, ${ }^{4}$ Margit Miesbauer, ${ }^{3}$ \\ Natalie Rotermund, ${ }^{5}$ Christian Lohr, ${ }^{5}$ Jörg Tatzelt, ${ }^{3,6}$ Konstanze F. Winklhofer, ${ }^{2,3,7,8}$ and Edgar R. Kramer ${ }^{1}$ \\ 1Development and Maintenance of the Nervous System, Center for Molecular Neurobiology, University Medical Center Hamburg-Eppendorf, Hamburg, Cermany. ${ }^{2}$ German Center for Neurodegenerative \\ Diseases, Munich, Germany. ${ }^{3}$ Neurobiochemistry, Adolf Butenandt Institute, Ludwig Maximilians University, Munich, Germany. ${ }^{4}$ Neonatal Screening/Metabolic Laboratory, Diagnostic Center, University \\ Medical Center Hamburg-Eppendorf, Hamburg, Germany. ${ }^{5}$ Division of Neurophysiology, Biocenter Grindel, University of Hamburg, Hamburg, Germany. ${ }^{6}$ Department of Biochemistry of Neurodegenerative \\ Diseases, Institute of Biochemistry and Pathobiochemistry, Ruhr University Bochum, Bochum, Germany. ${ }^{7}$ Munich Cluster for Systems Neurology, Munich, Germany. ${ }^{8}$ Department of Molecular Cell Biology, \\ Institute of Biochemistry and Pathobiochemistry, Ruhr University Bochum, Bochum, Germany.
}

\begin{abstract}
Parkin and the glial cell line-derived neurotrophic factor (GDNF) receptor RET have both been independently linked to the dopaminergic neuron degeneration that underlies Parkinson's disease (PD). In the present study, we demonstrate that there is genetic crosstalk between parkin and the receptor tyrosine kinase RET in two different mouse models of PD. Mice lacking both parkin and RET exhibited accelerated dopaminergic cell and axonal loss compared with parkin-deficient animals, which showed none, and RET-deficient mice, in which we found moderate degeneration. Transgenic expression of parkin protected the dopaminergic systems of aged RET-deficient mice. Downregulation of either parkin or RET in neuronal cells impaired mitochondrial function and morphology. Parkin expression restored mitochondrial function in GDNF/RET-deficient cells, while GDNF stimulation rescued mitochondrial defects in parkin-deficient cells. In both cases, improved mitochondrial function was the result of activation of the prosurvival NF-кB pathway, which was mediated by RET through the phosphoinositide-3-kinase (PI3K) pathway. Taken together, these observations indicate that parkin and the RET signaling cascade converge to control mitochondrial integrity and thereby properly maintain substantia nigra pars compacta dopaminergic neurons and their innervation in the striatum. The demonstration of crosstalk between parkin and RET highlights the interplay in the protein network that is altered in PD and suggests potential therapeutic targets and strategies to treat PD.
\end{abstract}

\section{Introduction}

Parkinson's disease (PD) is the most common movement disorder and is characterized by a progressive loss of midbrain dopaminergic (DA) neurons of the substantia nigra pars compacta (SNpc). The cause of this premature degeneration of DA neurons in patients with PD is not completely understood, but mitochondrial dysfunction, oxidative stress, protein aggregations, and reduced neurotrophic signaling are commonly observed in this context $(1$, 2). Rare familial forms of PD helped to define involved genes for example, genes encoding the proteins parkin and DJ-1 - and paved the way for the multiple-hit hypothesis for the more common sporadic PD cases. This multiple-hit hypothesis suggests that these accumulating alterations are finally leading to the collapse of the protein network essential for DA cell survival (3).

Recently, we discovered that mice that have genetically lost the glial cell line-derived neurotrophic factor (GDNF) receptor, the receptor tyrosine kinase RET, showed an age- and cell typespecific loss of SNpc DA neurons reminiscent of the histological

Conflict of interest: The authors have declared that no conflict of interest exists. Submitted: October 1, 2014; Accepted: February 12, 2015.

Reference information: J Clin Invest. 2015;125(5):1873-1885. doi:10.1172/JCI79300. alterations observed in patients with PD (4). Similarly, conditional Gdnf knockout (KO) mice were reported to show a strong degeneration phenotype in the DA system (5). Why the DA system depends on neurotrophic GDNF/RET signaling and which downstream signaling cascades are used for their beneficial effect is still unknown. In addition, we found that RET and DJ-1, a protein mutated in rare familial forms of $\mathrm{PD}$, are required to ensure $\mathrm{DA}$ cell body maintenance through the RAS/MAPK pathway (6).

The PD-associated gene PARK2 encodes the protein parkin, an E3 ubiquitin protein ligase important for mitochondrial integrity and quality control (7-9). Despite the manifold functions of parkin in cultured cells, none of the parkin KO mice show substantial DA system or severe behavioral abnormalities $(1,2)$. However, mice overexpressing wild-type parkin are protected against many neurodegenerative insults (10-12). We were interested in studying a possible crosstalk between parkin and RET in the DA system, since parkin and RET have been shown to function in the protein network altered in patients with PD $(13,14)$ and parkin influences intracellular signaling cascades of other receptor tyrosine kinases, such as the EGF receptor (15).

In this study, we accumulated evidence for a genetic crosstalk between parkin and RET in mice and identified a signaling cas- 
cade downstream of the RET receptor beneficial for mitochondrial integrity. Interestingly, enhanced parkin and GDNF/RET signaling can prevent mitochondrial defects caused by either RET or parkin deficiency in a mitophagy-independent manner. In lieu thereof, RET and parkin together protect mitochondrial morphology and function through the phosphoinositide-3-kinase/NF- $\mathrm{KB}$ (PI3K/ $\mathrm{NF}-\kappa \mathrm{B})$ pathway, which can prevent DA neuron degeneration in mice and likely in humans too.

\section{Results}

Late-onset substantia nigra-specific degeneration of DA neurons in mice lacking RET and parkin. To study the function of parkin in DA neurons of trophically impaired mice, we generated RET and parkin double-knockout (DKO) mice (herein referred to as RET/parkin DKO mice) by crossing parkin KO mice (16) with floxed Ret mice (Ret $t^{f / f l}$ mice) $(4,6,17,18)$ and an engineered dopamine transporter (Dat) promoter-driven Cre line (DCB-Cre mice) (DCB-Cre Ret ${ }^{f / / t}$ mice, herein referred to as RET KO mice) $(19,20)$. In viable and fertile RET/parkin DKO mice, no parkin or RET protein was detected in DA neurons of the SNpc or of the ventral tegmental area (VTA) (Figure 1, A and B, and Supplemental Figure 1, A and B; supplemental material available online with this article; doi:10.1172/ JCI79300DS1). As reported previously for only RET-deficient mice (4) and parkin $\mathrm{KO}$ mice $(16,21), 3$ - to 6-month-old RET/parkin DKO mice also showed an unchanged number of DA neurons in the SNpc and DA innervation in the striatum compared with agematched control mice (DCB-Cre mice), as seen by quantifying cells stained with antibodies against tyrosine hydroxylase $(\mathrm{TH})$, the rate-limiting enzyme for dopamine synthesis (Figure 1D, Supplemental Figure 1C, and Supplemental Figure 2, A and B). Parkin $\mathrm{KO}$ mice maintained an unchanged DA system during aging (ref. 16; Figure 1, E-G; Figure 2; Supplemental Figure 1, D-G; and Supplemental Figure 2, C-E), while 12- and 24-month-old RET KO and RET/parkin DKO mice lost $15 \%-21 \%$ and $20 \%-30 \%$ of the DA neurons in the SNpc and $33 \%-48 \%$ and $51 \%-56 \%$ of DA innervation in the dorsal striatum, respectively (Figure 1, E and F; Figure 2, A-C; Supplemental Figure 1, D and E; and Supplemental Figure 2, C and D). As reported previously for the RET-deficient mice (4), the DA cell loss in the SNpc of RET/parkin DKO mice was progressive over time (Figure $1 G$ ). The number of DA neurons in the VTA region was unaltered in all mouse lines, even during aging (Figure $1 \mathrm{H})$. In addition, the $\mathrm{G}$ protein-activated inward rectifier potassium channel-positive (GIRK2-positive) DA neurons $(22,23)$ - those that die in patients with PD - showed an increased loss in 24-month-old RET/parkin DKO mice (27\%) compared with that in RET KO mice (20\%) (Supplemental Figure 1, E-G). Quantification of DAT-stained DA terminals confirmed the reduced striatal DA innervation in RET KO and RET/parkin DKO mice (Figure 2, D and $\mathrm{E}$ ). Consistent with the striatal loss of DA innervation, we have also found a $19 \%$ decrease in total striatal dopamine in 1-year-old and a 30\% decrease in 2-year-old RET KO and RET/parkin DKO mice compared with that in control mice (DCB-Cre and/or Ret $t^{f / f l}$ mice) (Figure $2 \mathrm{~F}$ and Supplemental Figure 2E). The dopamine loss was also progressive over time in the RET/parkin DKO mice (Supplemental Figure 2F). Also the dopamine degradation product 3,4-dihydroxyphenylacetic acid (DOPAC) was significantly reduced in 2-year-old RET/parkin DKO mice (Figure 2G). However, we were not able to detect significant differences in TH protein levels or in activity-associated phosphorylated forms of $\mathrm{TH}$ in these aged mice (Supplemental Figure 3, B, C, H, and I; data not shown; and ref. 24). In addition, expression of the RET ligand GDNF and the RET coreceptors GFR $\alpha 1$ (for GDNF) and GFR $\alpha 2$ (for neurturin) as well as $\alpha$-synuclein, a protein upregulated in some patients with $\mathrm{PD}$, was not altered in the single and double KO mice (Supplemental Figure 3, B and H). To our knowledge, this trophically impaired environment revealed for the first time a DA system maintenance function of parkin in mice. In addition, progressive with age, only 24-month-old RET KO and RET/parkin DKO mice showed gliosis in the striatum and inflammation in the SNpc in comparison with DCB-Cre control mice, indicating a specific degeneration process (Supplemental Figures 4 and 5). In addition to the increase in gliosis and inflammation, the morphology of IBA1-stained microglia also changed in 24-month-old RET KO and RET/parkin DKO mice from resting to more bushy (Supplemental Figure 5, F and G). Furthermore, 1- and 2-year-old parkin KO, RET KO, and RET/parkin DKO mice show reduced horizontal activity in the open-field test as a first sign of a motor phenotype but no significant alterations in the pole and rotarod test (Figure 3, A, C, and D, and data not shown). In addition, the mutant mice showed increased anxiety, as they spent more time in the border zone in the open-field test (Figure 3B). In the elevated plus maze, they had fewer entries in the open arm, spent less time in the open arm, had fewer head dipping events, and reached the end of the open arm fewer times compared with DCB-Cre control mice (Figure 3, E-H). Increased anxiety is a symptom frequently observed in patients with $\operatorname{PD}(25,26)$.

Taken together, loss of parkin and RET in the DA system leads to histological, physiological, and behavioral alterations upon aging, which are similar to the clinical symptoms observed in PD.

RET and parkin deficiency leads to mitochondrial dysfunction and other cellular alterations. The genetic interaction between RET and parkin in the DA system of the RET/parkin DKO mice prompted us to search for more detailed morphological and subcellular alterations and underlying molecular mechanisms in DA neurons that could explain this in vivo crosstalk between RET and parkin. Despite the neurodegeneration phenotype, RET and parkin deletion did not significantly alter the activation levels of downstream signaling molecules, such as AKT (protein kinase B), ERK1 and ERK2, and S6 ribosomal protein, a canonical downstream target and marker for trophic signaling through the neuroprotective AKT/mTOR (mammalian target of rapamycin) signaling cascade, as quantified by Western blot detection of their phosphorylated forms (Supplemental Figure 3, B, D-H, J-M, and ref. 27). This might be due to compensatory mechanisms (28). Therefore, we included younger mice in our analysis to address the underlying mechanism of the observed RET-parkin crosstalk.

Interestingly, we found a significant reduction to $84 \%$ of the normal DA neuron cell size in 3- to 6-month-old and 24-month-old RET KO mice compared with that in DCB-Cre control mice but no alterations in parkin $\mathrm{KO}$ mice (Figure 4, A and $\mathrm{B}$ ). The cell size reduction is one of the first alterations found in RET KO mice, even before the neurodegeneration process has started, but it seems not to be more severe in RET/parkin DKO mice (Figure 4, A and B). This cell size reduction correlated also to a reduced cell capacity, as measured in the whole-cell patch-clamp configuration (Figure $4 \mathrm{C}$ ). 
A
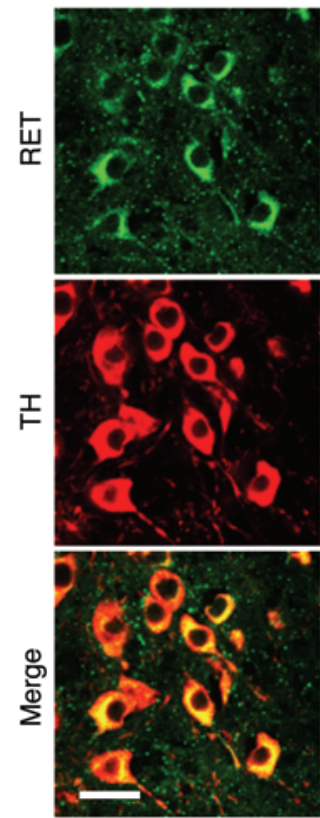
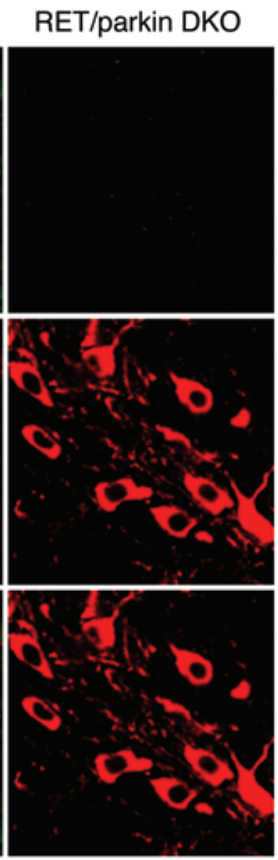
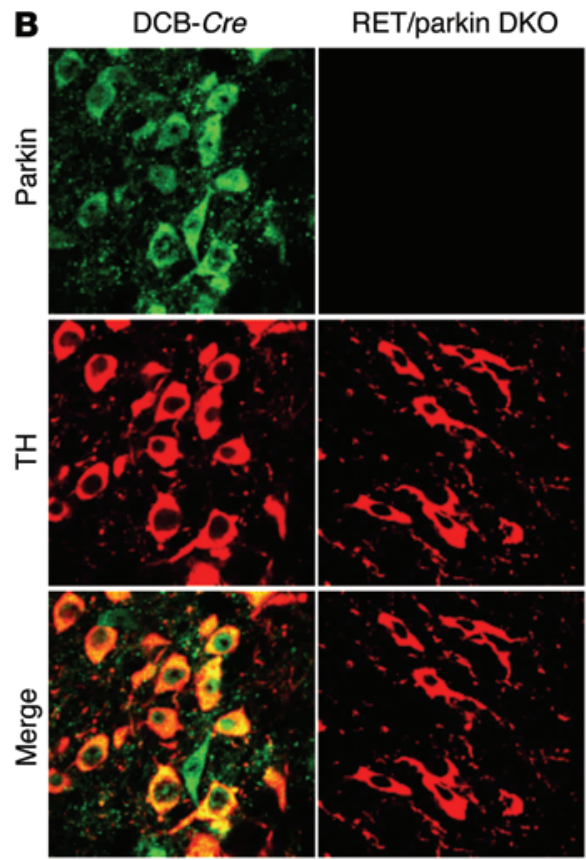

c

DCB-Cre

Parkin KO

RET KO

RET/parkin DKO
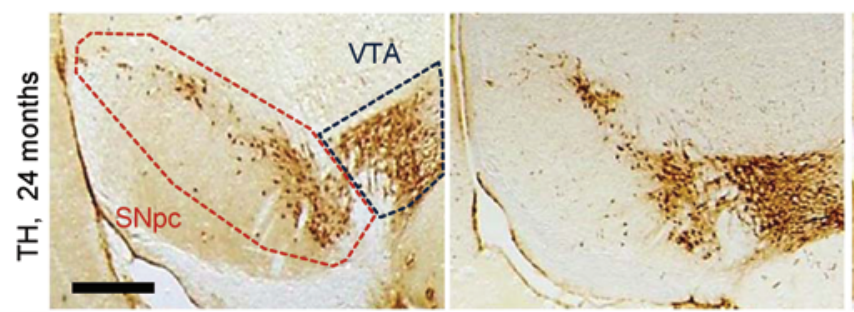

D $\mathrm{SNpc}$, 3-6 months

E SNpc, 12 months

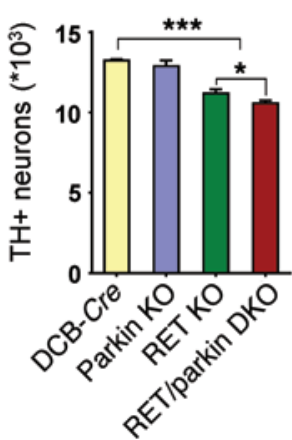

F $\mathrm{SNpc}, 24$ months

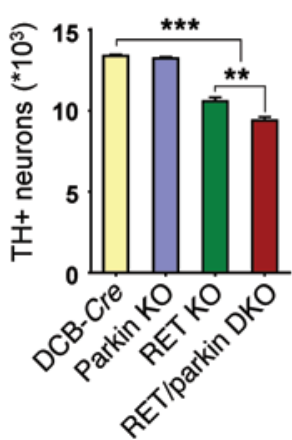

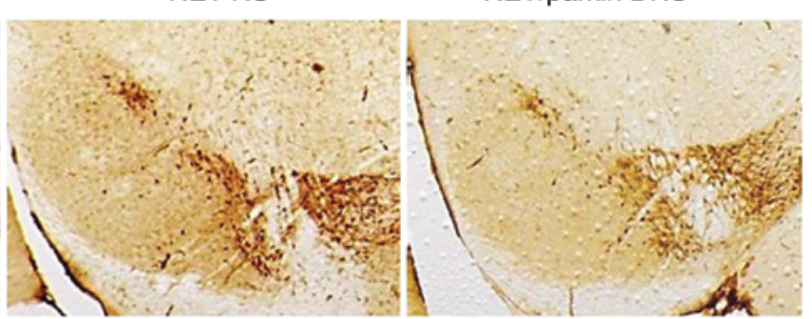

$\mathrm{SNpc}$

DCB-RET/ parkin DKO

H VTA, 24 months
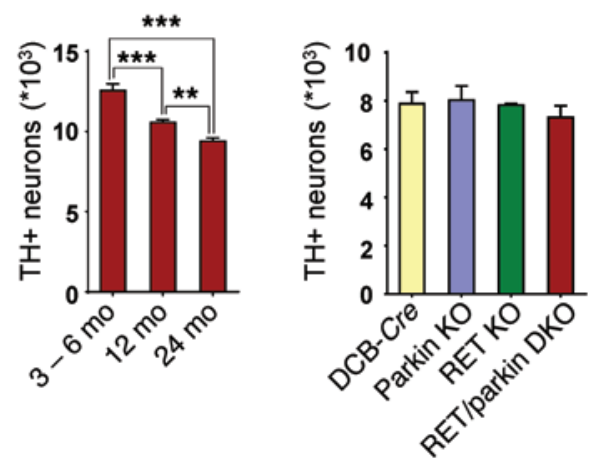

Figure 1. Late-onset and SNpc-specific degeneration of DA neurons in mice lacking RET and parkin. (A and B) Representative images of coronal sections from 3-month-old control DCB-Cre and RET/parkin DKO mice showing loss of (A) RET and (B) parkin expression in TH-stained DA neurons of the SNpc in the RET/parkin DKO mice (scale bar: $25 \mu \mathrm{m}$ ). (C) Representative images of coronal midbrain sections from 24-month-old mice with the indicated genotypes showing DA neurons in the SNpc and VTA stained with TH antibody (scale bar: $250 \mu \mathrm{m}$ ). (D-H) Stereological quantification of TH-positive neurons in the SNpc of (D) 3- to 6-month-old ( $n=3-5)$, (E) 12-month-old $(n=3-6)$, and (F) 24-month-old $(n=3-6)$ mice. (G) Progressive and age-dependent loss of SNpc DA neurons in RET/parkin DKO mice $(n=5-6)$ over time. Data were obtained from $\mathbf{D - F}$. $(\mathbf{H})$ TH-positive neurons were not lost in the VTA region in any of the 24-month-old mice $(n=3-6)$. Data are represented as mean \pm SEM. ${ }^{*} P \leq 0.05$, ${ }^{* *} P \leq 0.01,{ }^{* * *} P \leq 0.001$, 1-way ANOVA, Newman-Keuls post-hoc test.

However, the kinetics of action potentials in SNpc DA neurons were not altered in the mutant mice (Supplemental Figure 6). Next, we analyzed the total dopamine levels in 3- to 6-month-old mice and found a significant reduction only in the RET/parkin DKO mice compared with the DCB-Cre control mice (Figure 4D), even without detectable DA cell or innervation loss (Figure 1D and Supplemental Figure 2, A and B). This suggests a physiological role for RET and parkin in regulating dopamine levels already in young mice and 
A

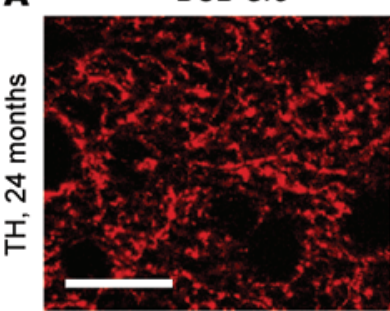

B Dorsal striatum 12 months

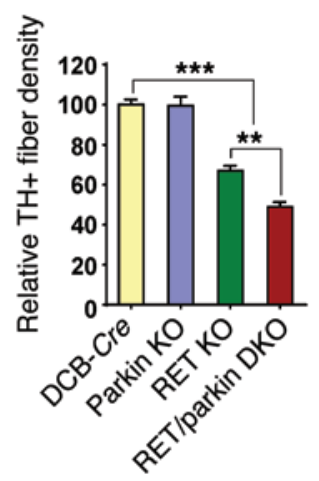

D

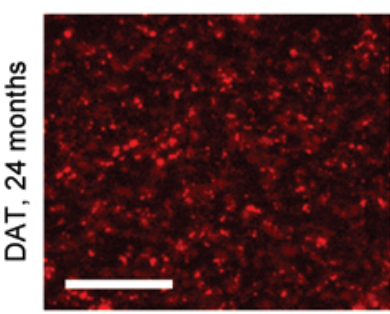

E

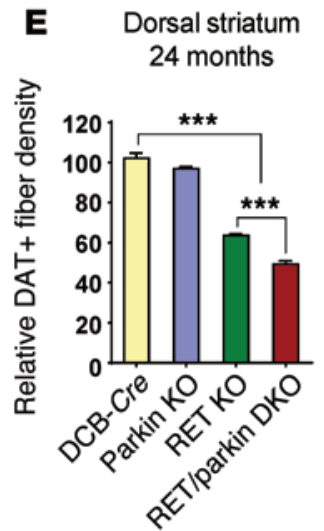

$\mathbf{F}$
Parkin KO

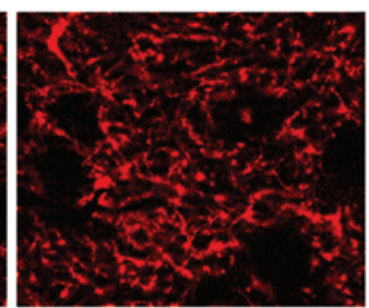

Dorsal striatum

24 months

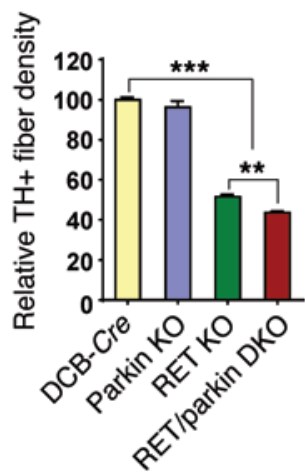

Parkin KO

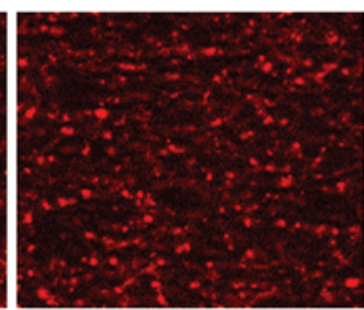

Striatum, 24 months

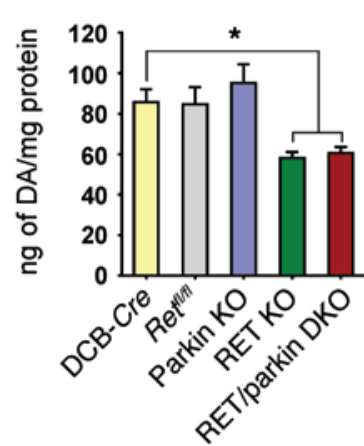

RET KO
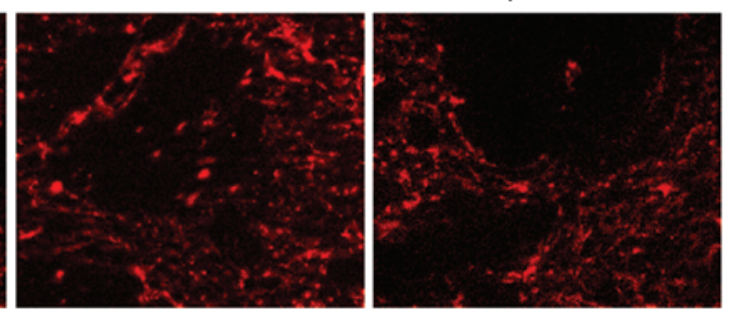

RET KO

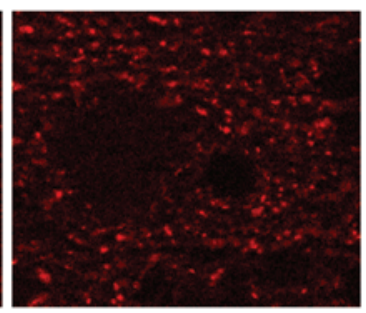

G Striatum, 24 months

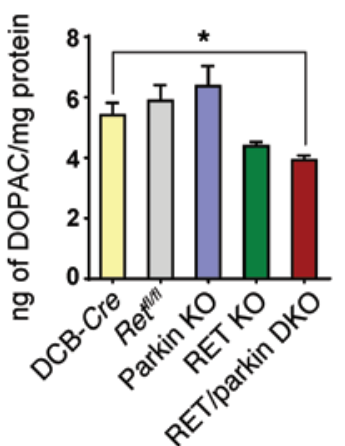

Figure 2. RET and parkin maintain DA innervation of the striatum. (A) Representative images of coronal sections with immunofluorescent staining of TH fibers in the dorsal striatum of 24-month-old mice of the indicated genotypes (scale bar: $10 \mu \mathrm{m}$ ). (B and C) Quantification of TH fiber density in dorsal striatum of (B) 12-month-old mice $(n=4)$ and (C) 24-month-old mice $(n=3-4)$. (D) Representative images of coronal sections with fluorescent DAT staining of DA fibers in the dorsal striatum of 24-month-old mice of the indicated genotypes (scale bar: $10 \mu \mathrm{m}$ ). (E) Quantification of DAT fiber density in the dorsal striatum of 24-month-old mice $(n=3-4)$. (F and $\mathbf{G})$ Striatal measurements of $(\mathbf{F})$ total dopamine and $(\mathbf{G})$ total DOPAC levels in 24-month-old mice of the indicated genotypes $(n=4-7)$. Data are represented as mean $\pm S E M$. ${ }^{* *} P \leq 0.01,{ }^{* *} P \leq 0.001,1$-way ANOVA, Newman-Keuls post-hoc test for $\mathbf{B}, \mathbf{C}$, and $\mathbf{E}$; ${ }^{*} P \leq 0.05$, unpaired 2-tailed $t$ test for $\mathbf{F}$ and $\mathbf{G}$.

not only during the neurodegeneration process in old mice, without altering TH and DAT protein levels (Supplemental Figure 1A).

Since PD is associated with mitochondrial dysfunction and parkin has been shown to be a regulator of mitochondrial integrity required for adequate energy production $(7,8)$, we had a closer look at the mitochondrial function and energy supply in the DA system of our mouse mutants. Indeed, when we measured the total ATP levels in SNpc tissue lysates, we observed a $20 \%$ reduction in adult RET/ 


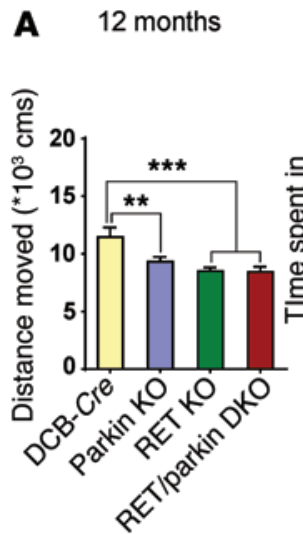

B 12 months
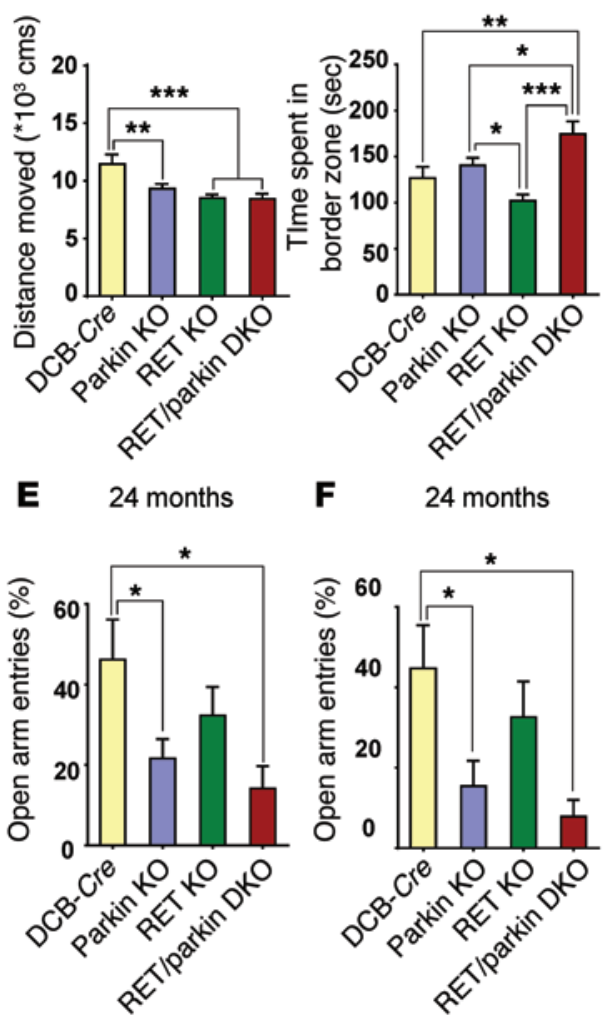

C
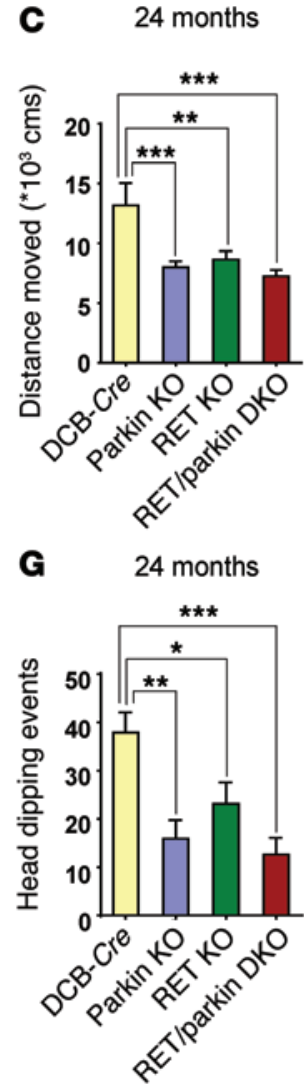

D

12 months

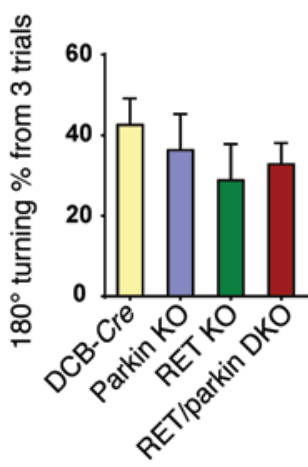

H

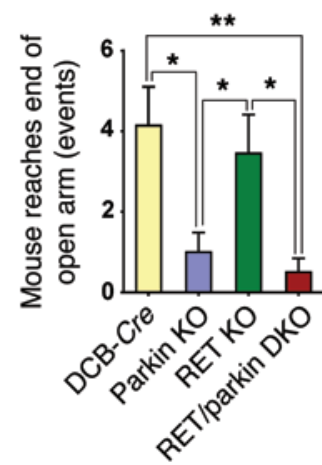

Figure 3. Aged parkin- and RET-deficient mice exhibit behavioral alterations. (A-C) Open-field test for measuring horizontal activity and anxiety in aged mice is shown. Distance moved (in $\mathrm{cm}$ ) during a 10-minute duration in (A) 12-month-old ( $n=11-24)$ and (C) 24-month-old ( $n=6$-17) mice of the indicated genotypes is shown. (B) Thigmotactic behavior was measured by analyzing the time (in seconds) spent by each mouse in the border zone of the arena during the first 5 minutes of a 10-minute trial of 12-month-old mice $(n=11-24)$ of the indicated genotypes. (D) Quantification showing $180^{\circ}$ turning behavior (\%) from 3 trials of a pole test in 12 -month-old mice $(n=5-13)$. (E-H) Quantification of anxiety behavior from 24-month-old mice during 5 minutes on an elevated plus maze of the indicated genotypes $(n=7-11)$. (E) Open arm entries, (F) open arm duration, (C) head dipping events, and $(\mathbf{H})$ number of times mouse reaches the end of an open arm are shown. Data are represented as mean \pm SEM. ${ }^{*} P \leq 0.05,{ }^{* *} P \leq 0.01,{ }^{* * *} P \leq 0.001,1$-way ANOVA, Newman-Keuls post-hoc test.

parkin DKO mice but not in single KO mice compared with DCB-Cre control mice (Figure $4 \mathrm{E}$ ). We confirmed this finding in a cellular system using siRNAs against RET, parkin, or both in TH-expressing SH-SY5Y cells. The siRNA approach downregulated parkin and RET mRNA levels by $70 \%$ to $80 \%$ (Supplemental Figure 9, A and B), resulting in a 20\% reduction of total ATP levels in the single RET and parkin knockdown cells and a $40 \%$ reduction in the RET/parkin double knockdown cells (Figure 4F). Interestingly, treating parkin-deficient SH-SY5Y cells with the RET ligand GDNF and its coreceptor GFR $\alpha 1$ resulted in the complete rescue of the ATP levels (Figure 4G) without altering parkin mRNA levels (Supplemental Figure 9C).

The mitochondria-enriched fractions from the SNpc of adult RET/parkin DKO mice showed a highly significant $20 \%$ reduction of complex I activity compared with that in DCB-Cre control mice but no alteration in single-deficient mice (Figure $4 \mathrm{H}$ ). In addition, the reduction of complex I activity seemed progressive and was significant in 12-month-old RET KO mice (Figure 4I). In SH-SY5Y cells with single and double knockdown of RET and parkin, we also observed around 20\% reduction of the complex I activity (Supplemental Figure 9D). Investigating the expression level of mitochonneurons) (Figure 5B). The wt h-parkin mice overexpressed parkin in DA cell bodies of the midbrain 7- to 8-fold compared with the amount of endogenous parkin protein and 3- to 4-fold in the DA innervation field of the striatum (Supplemental Figure 7, A-C). Next, we crossed the parkin transgenic mice with the RET KO mice (RET KO/wt h-parkin mice) and analyzed the single- and doublemutant mice at the age of 12 months, when the RET KO mice already showed significant histological and physiological changes (Figures 1-4 and Supplemental Figures 1-3). The single parkin transgenic mice did not show alterations compared with $\operatorname{Ret}^{A / / A}$ control mice in all parameters analyzed (Figure 5, C-G, and Supplemental Figure 7, D-I). Surprisingly, the parkin transgenic overexpression was able to prevent the age-dependent DA cell body loss in the SNpc of RET KO mice (Figure 5C and Supplemental Figure 7D). Transgenic overexpression of human parkin in wild-type or RET KO mice did not alter VTA DA neuron numbers (Figure 5D and Supplemental Figure 7E). Parkin overexpression in the RET KO mice largely prevented loss of DA innervation and led to nearly normal total dopamine levels in the striatum (Figure 5, E and F, and Supplemental Figure 7, F-I). Thus, overexpression of parkin seems to be neuroprotective in trophically 
A $\mathrm{SNpc}, 24 \mathrm{mo}$

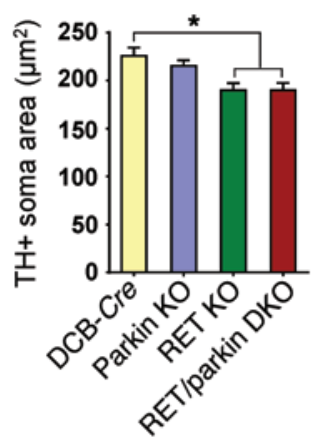

F

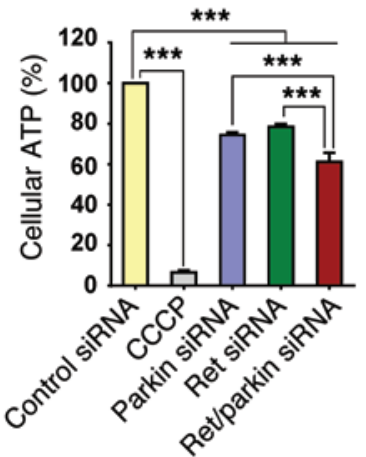

B SNpc, 3-6 mo

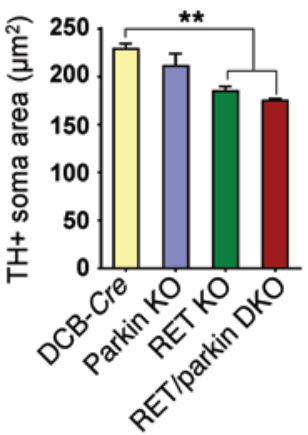

G

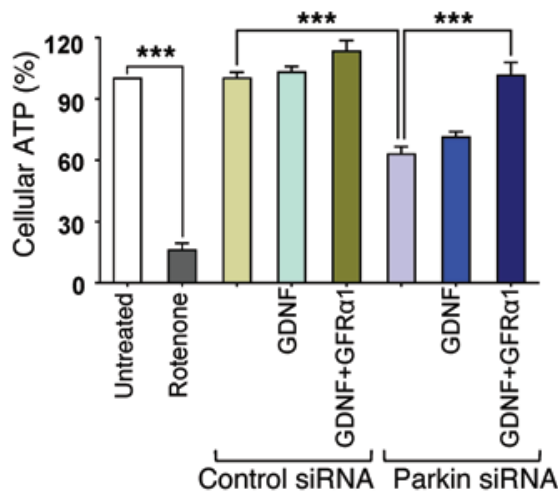

SH-SY5Y cells
C SNpc, 3-6 mo

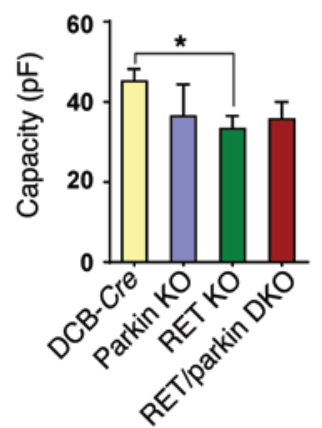

H

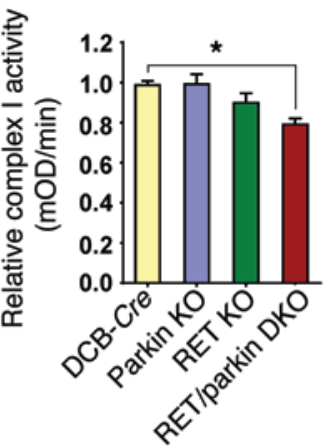

Striatum, 3-6 mo

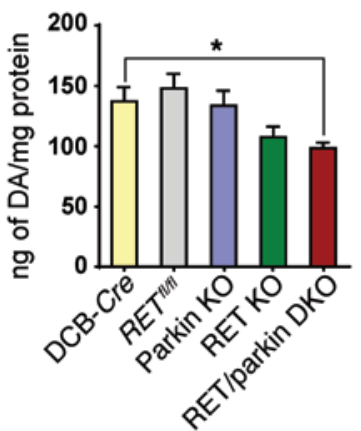

SNpc, $3-6$ mo

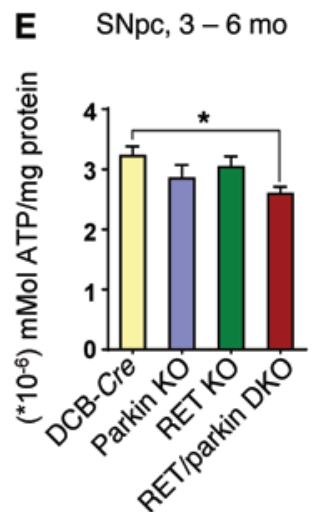

I SNpc, $12 \mathrm{mo}$

Figure 4. Cellular changes in the RET/parkin DKO mice. (A and B) Cell soma area measurements of SNpc TH-positive neurons in (A) 24-month-old and (B) 3- to 6-month-old mice $(n=3)$. (C) Membrane capacitance of GFP-positive neurons from $n=5-7$ cells per genotype were measured. (D) HPLC measurements of total striatal dopamine levels in 3- to 6-month-old mice $(n=5-7)$. (E) Measurements of total cellular ATP levels in the SNpc of 3-to 6-month-old mice $(n=5)$. (F) Measurements of cellular ATP levels in the SH-SY5Y cells after treatment with parkin and RET siRNA or dissipation of the mitochondrial membrane potential by the uncoupler carbonyl cyanide m-chlorophenylhydrazone (CCCP) ( $n=4$ experiments). (G) Measurements of ATP levels in the SH-SY5Y cells treated with parkin siRNA and/or with GDNF and GFR $\alpha 1$ ( $n=3$ experiments). Rotenone, which interferes with electron transport chain, was used as positive control. (H) Mitochondrial complex I activity measurements in the SNpc of 3- to 6-month-old mice $(n=6-9)$. (I) Mitochondrial complex I activity measurements in the SNpc of 24 -month-old mice $(n=5)$. Data are represented as mean \pm SEM. ${ }^{*} P \leq 0.05,{ }^{* *} P \leq 0.01,{ }^{* * *} P \leq 0.001,1$-way ANOVA, Newman-Keuls post-hoc test for $\mathbf{A}, \mathbf{B}, \mathbf{D}$, and $\mathbf{F}-\mathbf{H} ;{ }^{*} P \leq 0.05,1$-way ANOVA, unpaired 2-tailed $t$ test for $\mathbf{C}$, E, and $\mathbf{I}$.

impaired mice and can prevent age-dependent DA cell body and innervation loss. Interestingly, also the cell size reduction in RET KO mice can be prevented by overexpressing human parkin (Figure $5 G$ ).

The modulatory role of parkin on the RET loss-of-function phenotype, together with their combined influence on the mitochondrial energy supply, supports the idea of a genetic and functional crosstalk between RET and parkin and raises the question about the molecular mechanism and cellular signaling events.

Convergent signaling pathways of RET and parkin regulate mitochondrial morphology. Next, we investigated the mechanism of how RET and parkin influence mitochondrial function. Acute silencing of parkin and PINK1 in cultured cells (30-32) has previously been shown to increase mitochondrial fragmentation with ultrastructural damage of cristae (reviewed in ref. 33). Therefore, we first investigated the mitochondrial morphology in our mouse models. We used TH-labeled SNpc sections from 24-month-old DCB-Cre, RET KO, parkin KO, and RET/parkin DKO mice for an electron microscopic study to specifically visualize the morphology of mitochondria in DA neurons (Supplemental Figure 8, A and B). The total number of mitochondria or damaged mitochondria per
$100 \mu \mathrm{m}^{2}$ area cytoplasm was not altered, but we observed a reduction of the mitochondrial size in parkin KO and RET/parkin DKO mice compared with that in littermate controls (Supplemental Figure $8, \mathrm{C}-\mathrm{E})$. Although these data show a trend toward ultrastructural alterations of mitochondria in parkin-deficient mice - well documented for DJ-1-deficient mice (34) but not for parkin- or PINK1-deficient mice (30-32) - this does not shed light on the RET-parkin crosstalk mechanism on mitochondrial function.

It has recently been shown that neither increased nor decreased RET signaling has an impact on PINK1/parkin-induced mitophagy (35). In line with this finding, we did not observe changes in autophagy-related proteins, such as p62 and LC3, or alterations in the AKT/mTOR pathway in RET KO, parkin KO, and RET/parkin DKO mice (Supplemental Figure 3, B-H, and refs. 7, 36). Moreover, severe stress conditions that seem to be required for parkin-induced mitophagy are unlikely to occur in our mouse models, since we did not find evidence for increased oxidative stress (Supplemental Figure 8F and ref. 31). Thus, the effect of RET signaling on mitochondrial integrity cannot be explained by modulating parkin-induced mitophagy. 
A
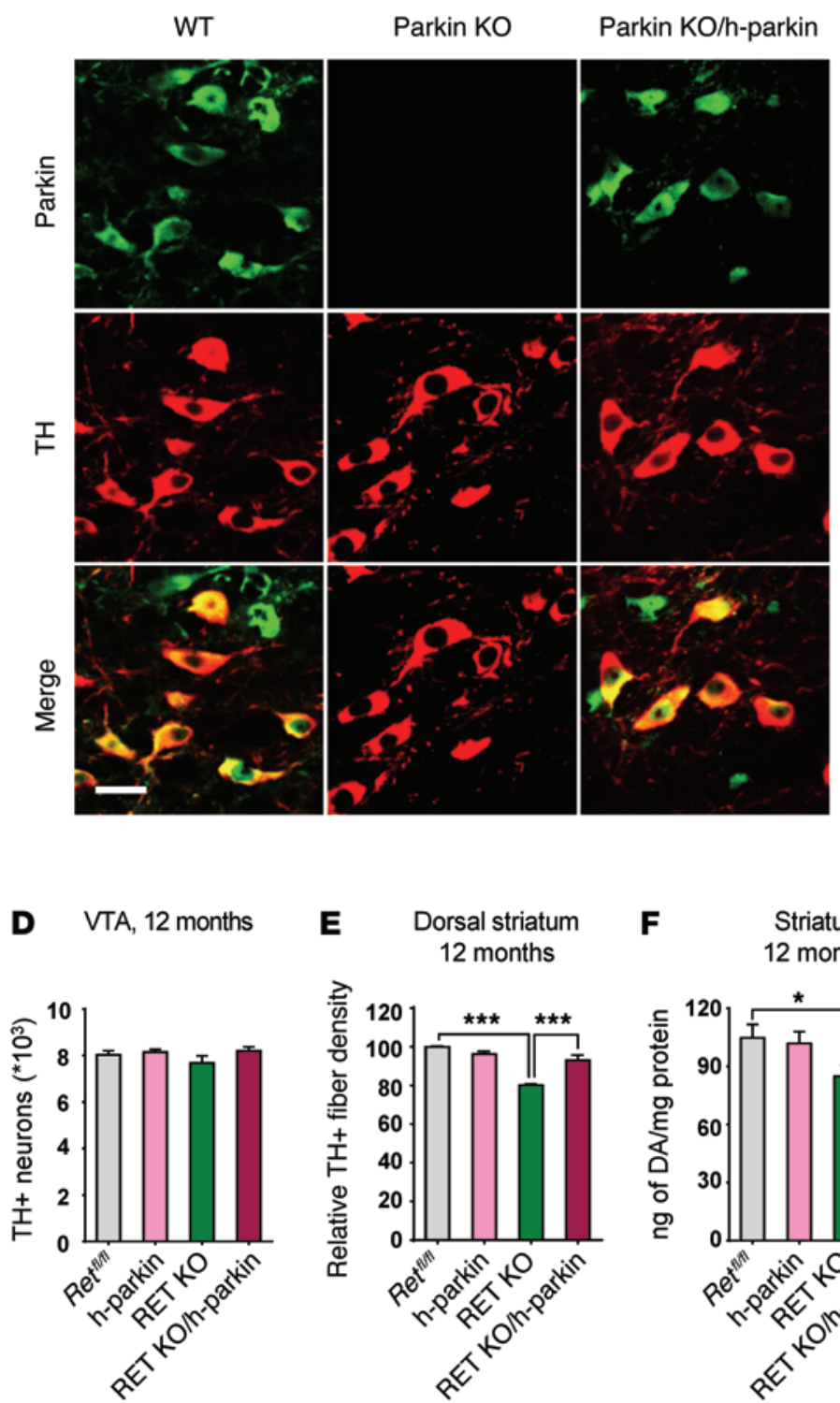

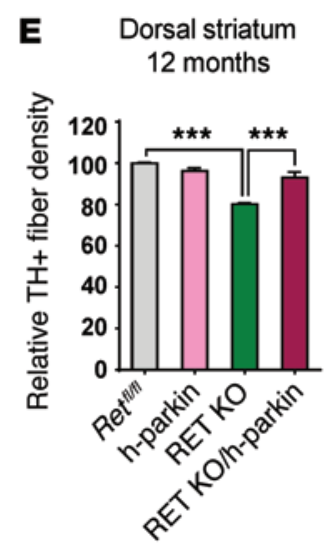

B

SNpc and VTA

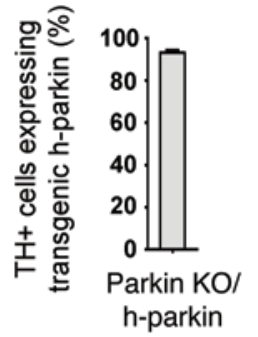

C

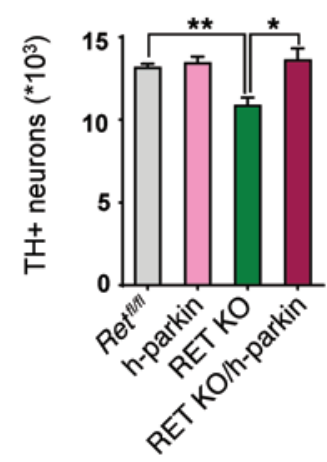

$\mathbf{F}$

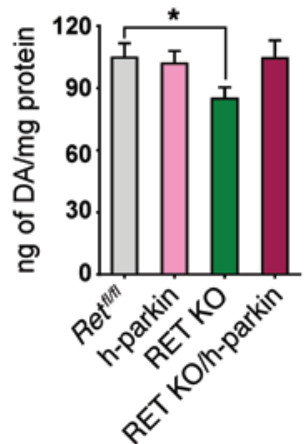

G

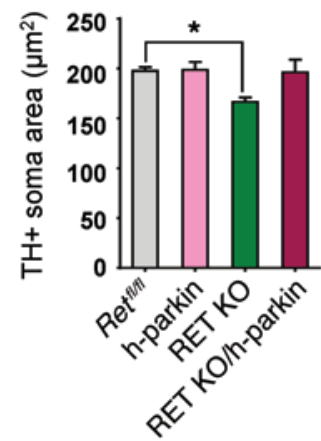

Figure 5. Transgenic parkin prevents neurodegeneration in RET KO mice. (A) Expression of human parkin (h-parkin) in $\mathrm{DA}\left(\mathrm{TH}^{+}\right)$neurons of the SNpc in transgenic mice crossed with parkin KO mice. Wild-type and transgenic mice but not parkin KO mice show parkin expression (scale bar: $25 \mu \mathrm{m}$ ). (B) Cell counting revealed that $93 \%$ of TH-positive cells express human parkin in the SNpc and VTA region ( $n=3,150-200$ cells counted per mouse). (C and D) Stereological quantification of $\mathrm{TH}$-positive neurons in (C) SNpc and (D) VTA of 12-month-old mice of the indicated genotypes $(n=3-4)$. (E) TH fiber density in dorsal striatum of 12-month-old mice of the indicated genotypes $(n=3-5)$. (F) Striatal HPLC measurements of total dopamine levels in 12-month-old mice $(n=4-7)$. (G) Cell soma area measurements of SNpc THpositive neurons in 12-monthold mice $(n=3)$. Data are represented as mean $\pm \mathrm{SEM}$. ${ }^{*} P \leq 0.05,{ }^{* *} P \leq 0.01$, ${ }^{* *} P \leq 0.001,1$-way ANOVA, Newman-Keuls post-hoc test for $\mathbf{C}-\mathbf{E}$; ${ }^{*} P \leq 0.05$, unpaired 2-tailed $t$ test for $\mathbf{F}$ and $\mathbf{G}$
Under moderate stress conditions with only minor mitochondrial defects, parkin has been shown to activate the prosurvival NF- $\mathrm{kB}$ pathway, which can upregulate the mitochondrial GTPase OPA1, which promotes mitochondrial inner membrane fusion, maintains cristae integrity, and prevents cytochrome $c$ release under apoptotic stress $(37,38)$. Since we could envision mild cellular stress conditions in RET- and parkin-deficient mice, we addressed the question of whether the NF- $\mathrm{BB}$ pathway might contribute to the effects of RET signaling on mitochondrial morphology. The mitochondrial network in SH-SY5Y cells was visualized by using antibodies against the outer membrane protein TOM20, and mitochondria were classified into tubular or fragmented based on their length (Figure 6A). As shown previously (39), treatment of SH-SY5Y cells with parkin-specific siRNA increased mitochondrial fragmentation to $70 \%$ to $85 \%$ compared with $25 \%$ to $40 \%$ in control siRNA-treated cells (Figure 6, B, C, and E). Interestingly, the mitochondrial fragmentation phenotype in SH-SY5Y cells due to parkin silencing could be completely rescued by treating the cells at the same time with the RET receptor ligand GDNF and its coreceptor GFR $\alpha 1$ (Figure 6, B, C, and E). Notably, the GDNF/GFR 1 treatment had no effect on parkin protein levels (Figure 6D), and parkin siRNA did not alter RET mRNA levels (Supplemental Figure 9B).

To get insight into the mechanism underlying the rescue activity of GDNF/GFR $\alpha 1$, we specifically inhibited signaling pathways that can be activated by RET. The GDNF/GFR $\alpha 1$-dependent rescue of the mitochondrial fragmentation phenotype in parkin knockdown cells could be blocked by incubating the cells with the PI3K inhibitor LY294002 but not with the mitogen-activated protein kinase kinase inhibitors, such as the MAPK/ERK kinase 1 (MEK1) inhibitor PD98059 or the MEK1+2 inhibitor U0126 (Figure 6, B and C). These inhibitors (LY294002, PD98059, and U0126) per se had no effect on mitochondrial morphology or parkin mRNA levels (Supplemental Figure 9, E and F). Interestingly, activation of the NF-kB pathway by overexpressing IKK $\beta$ also rescued the mitochondrial fragmentation phenotype of parkin-depleted SH-SY5Y cells (Figure 6E). In addition, the increased expression of the dominant-negative NF- $\mathrm{KB}$ 
A
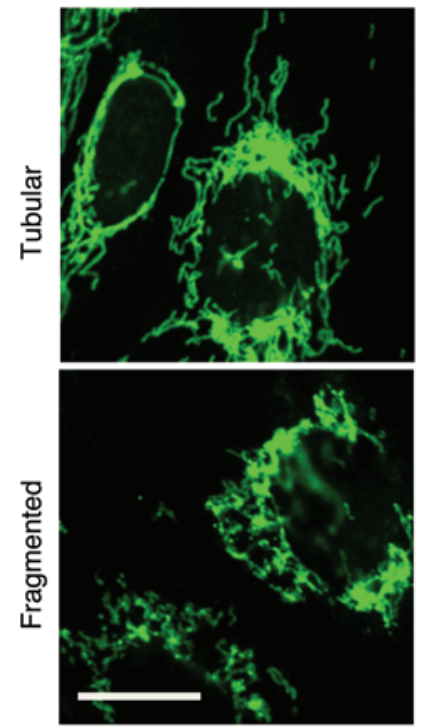

C

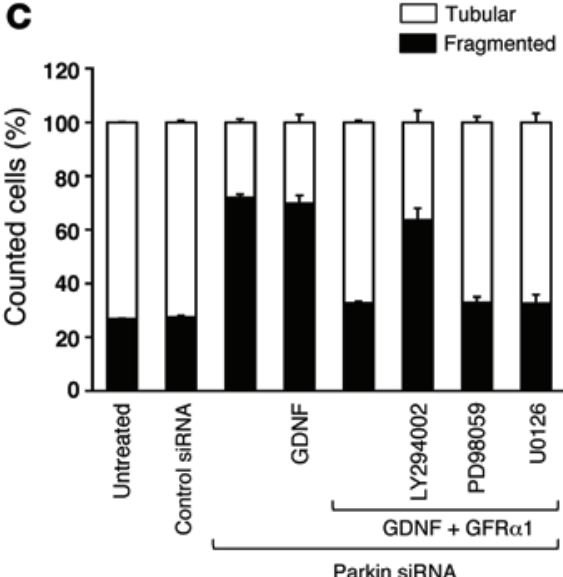

B

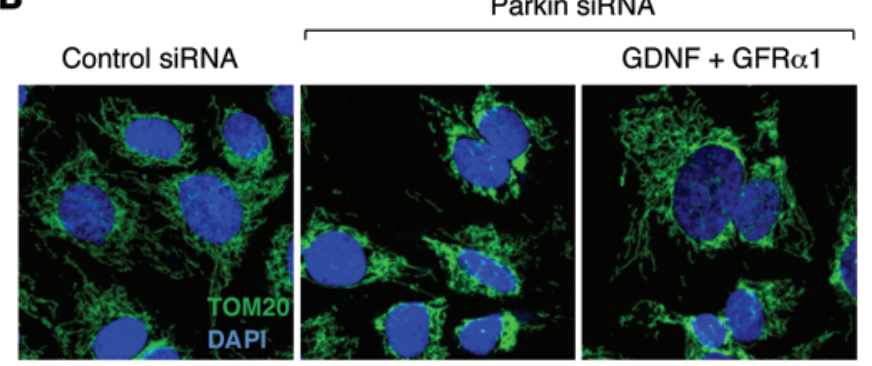

Parkin siRNA + GDNF + GFR 1

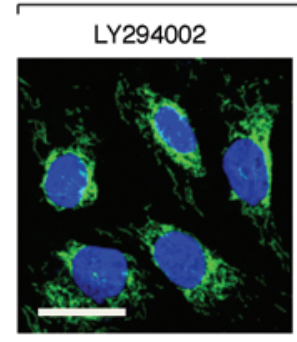

D

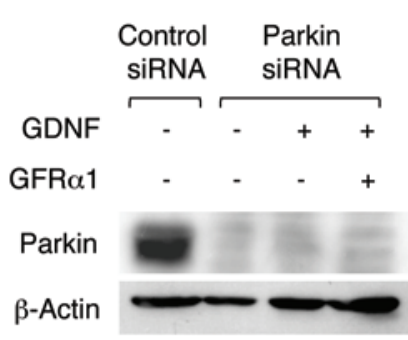

E
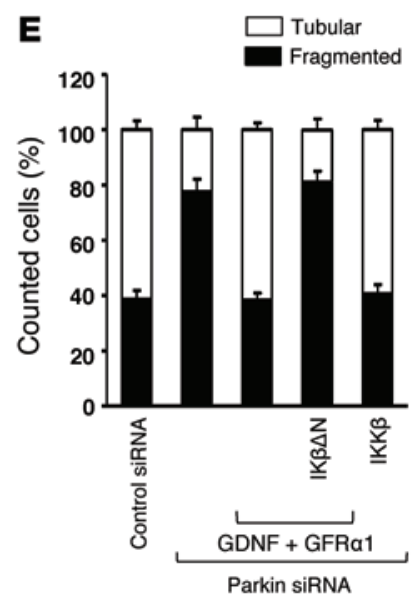

Figure 6. Mitochondrial fragmentation in parkin knockdown SH-SY5Y cells can be prevented by GDNF and GFR 1 . (A) Representative images of tubular (normal) and fragmented (damaged) mitochondria in cells immunofluorescently labeled with TOM20 antibody (scale bar: $10 \mu \mathrm{m}$ ). (B) Representative images of TOM20-stained mitochondria (green) and DAPI-stained nuclei (blue) in cells after transfection with control or parkin siRNA and additional treatment with GDNF and GFR 1 and the inhibitors LY294002, PD98059, or U0126 as indicated (scale bar: $10 \mu \mathrm{m}$ ). (C) Quantification of cells with tubular or fragmented mitochondria from B. (D) Western blot showing the efficient knockdown of parkin protein in cells after parkin siRNA transfection, independent of further treatment with or without GDNF and GFR $\alpha$; $\beta$-actin is shown as a loading control. (E) Quantification of cells with tubular or fragmented mitochondria from Supplemental Figure $8 \mathrm{~A}$ treated with parkin or control siRNA. Some cells were additionally stimulated with GDNF and GFR $\alpha 1$ and transfected with IK $\beta \Delta N$ (dominant-negative NF-KB signaling inhibitor) or IKK $\beta$ (NF-KB activator) constructs. Data are represented as mean \pm SEM; $n=3$ experiments.

inhibitor IK $\beta \Delta \mathrm{N}$ completely blocked the rescuing effect of GDNF/ GFR $\alpha 1$ in this context (Figure 6E and Supplemental Figure 10A). Thus, GDNF/GFR $\alpha 1$ seems to signal via the PI3K and NF- $\kappa$ B pathway to restore the mitochondrial network in parkin-deficient cells.

An expression analysis of mitochondrial proteins by Western blotting in GDNF-treated SH-SY5Y cells and in parkin- and RET-deficient mice did not support a role of GDNF/RET signaling on mitochondrial biogenesis (Supplemental Figure 3 and Supplemental Figure 10, B and C).

Strikingly, our experiments revealed an influence of endogenous RET on the morphology of mitochondria (Figure 7). Silencing of RET by siRNA in SH-SY5Y cells not only doubled the amount of cells with fragmented mitochondria but also led to a condensation of the mitochondrial network around the nucleus in more than $20 \%$ of cells (Figure 7, A-C). This phenotype was specific for the loss of RET protein kinase activity, since we could rescue the mitochondrial morphology defects in these cells by overexpressing human wild-type RET but not a RET kinase-dead mutant (RET KiD) (Figure 7C). Furthermore, we could rescue the mitochondrial RET knockdown phenotype by overexpression of parkin (Figure 7C). Notably, the increased expression of parkin had no effect on RET mRNA or protein levels (Figure 7D). Overexpression of RET, RET KiD, or parkin in control siRNA-treated cells had no effect on the mitochondrial morphology, confirming the specific rescue function of these constructs in the RET knockdown context (Supplemental Figure 10D). Both, RET and parkin activated the same signaling components - PI3K and NF- $\mathrm{KB}$ - to preserve a tubular mitochondrial network (Figure 7E). Overexpression of wild-type PI3K or a membrane-anchored constitutively active form of PI3K (PI3Kmyr) could markedly rescue the RET knockdown phenotype 

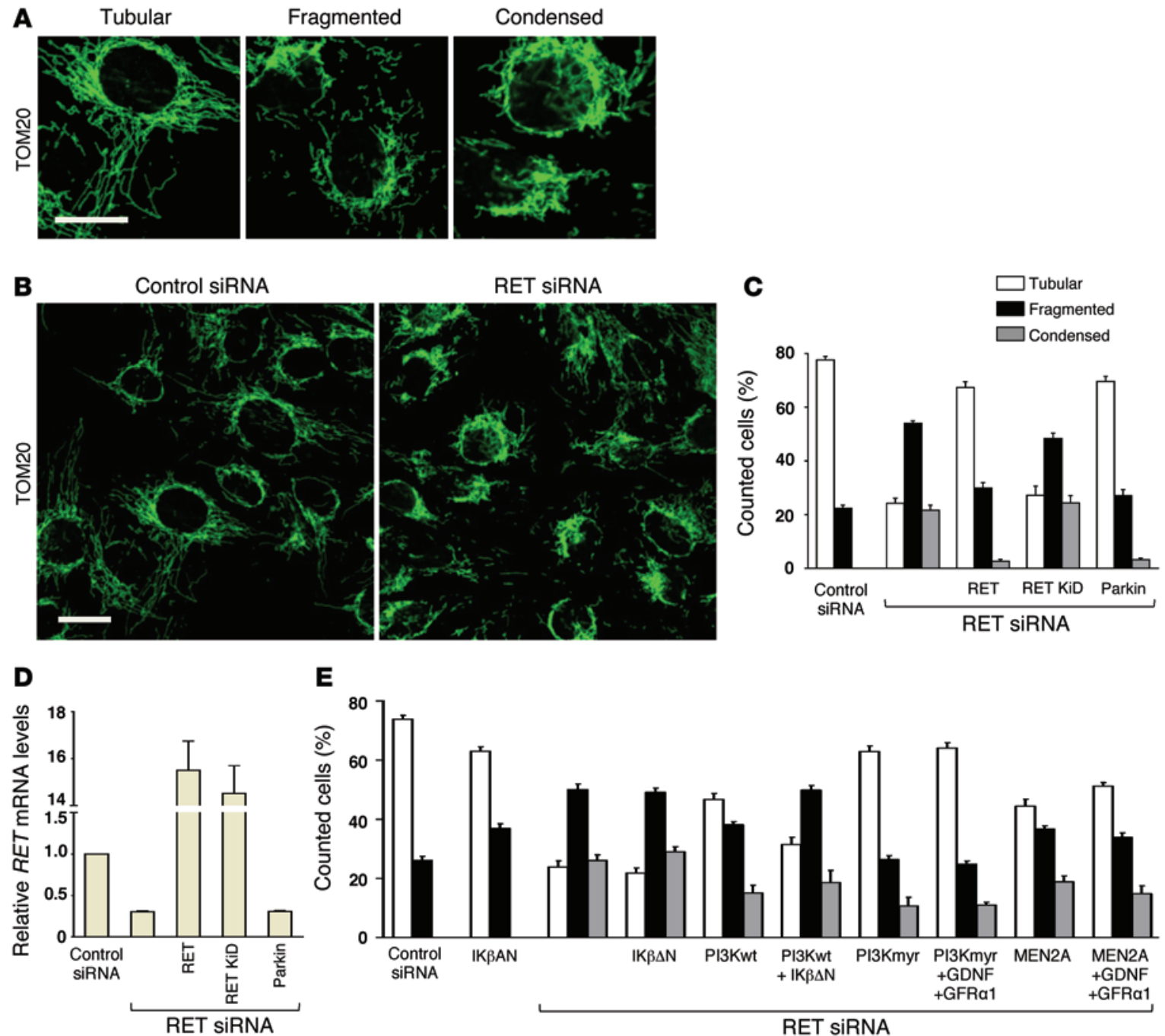

Figure 7. RET knockdown in SH-SY5Y cells induces mitochondrial network alterations. (A) Representative images of tubular (normal), fragmented (damaged), and condensed (damaged) mitochondria labeled with TOM2O antibody in SH-SY5Y cells (scale bar: $10 \mu \mathrm{m}$ ). (B) Representative images of TOM20-labeled mitochondria in cells transfected with control or RET siRNA (scale bar: $10 \mu \mathrm{m}$ ). (C) Quantification of cells with tubular, fragmented, or condensed mitochondria after transfection with control or RET siRNA. Some cells were additionally transfected with wild-type RET, kinase-dead RET (RET KiD), or parkin constructs. (D) Relative RET mRNA levels quantified by RT-PCR in the cell samples analyzed in C. (E) Quantification of tubular, fragmented, or condensed mitochondria in cells treated with RET siRNA and/or overexpressing IK $\beta \Delta N$, PI3Kwt, constitutive active PI3Kmyr, or MEN2A in the presence or absence of GDNF and GFR $\alpha 1$ in the culture medium. Data are represented as mean $\pm \mathrm{SEM} ; n=3$ experiments. Newman-Keuls multiple-comparison test, performed after 2-way ANOVA analysis, was performed for the data in $\mathbf{C}$ and $\mathbf{E}$ (see Supplemental Tables 3 and 4).

(Figure 7E). However, this was not the case when the NF-kB pathway was blocked by IK $\beta \Delta \mathrm{N}$ overexpression (Figure $7 \mathrm{E}$ ). Treating RET knockdown cells with GDNF/GFR $\alpha 1$ in addition to overexpressing PI3Kmyr or a constitutively active form of RET (MEN2A) did not enhance the rescuing effect (Figure 7E). Taken together, this argues for a role of GDNF/GFR $\alpha 1 /$ RET and parkin on maintaining mitochondrial morphology via the PI3K/NF- $\mathrm{kB}$ pathway.

\section{Discussion}

In the present study, we report that deletion of parkin and RET in mice leads to an enhanced neurodegeneration phenotype, with increased SNpc DA cell body and innervation loss, when compared with mice that only lack RET. Conversely, transgenic overexpression of parkin prevented the neurodegeneration phenotype of RET KO mice. Both parkin signaling and GDNF/RET signaling are required for normal mitochondrial function and morphology. Mitochondrial integrity as a novel molecular target of GDNF/RET signaling can help to explain the RET loss-of-function phenotype in the DA system. Overexpression of parkin or activation of GDNF/RET signaling can rescue the mitochondrial loss-of-function phenotype of RET or parkin through the PI3K/NF- $\mathrm{KB}$ pathway, respectively. Together, these data demonstrate that parkin cooperates with GDNF/RET signaling to prevent neurodegeneration of the DA system in mice and to maintain proper mitochondrial function (Supplemental Figure 11).

By establishing a new DA neuron-specific RET KO mouse, we have confirmed the previous observation that RET deficiency in mice leads to a neurodegeneration of DA neurons (4). First, in these RET KO mice, we found a reduced DA soma size and partially reduced dopamine levels in the striatum (age 3 months); later, DA fibers were lost and DA cell bodies died, which correlates with 
behavioral alterations (age 1-2 years). Finally, inflammation in the $\mathrm{SNpc}$ and gliosis in the striatum were observed (age 2 years), consistent with a progressive dying-back process postulated for PD (3). Interestingly, the constitutive parkin-deficient mice seem to reveal the cell survival function of parkin only under additional stress conditions, here generated by the absence of the neurotrophic GDNF/ RET signaling cascade. This might be explained by compensatory mechanisms in the classical parkin KO mice that maintain the survival signal in DA neurons. Upon deletion of parkin in addition with the RET receptor, the signaling intensity of the PI3K/NF- $\mathrm{BB}$ survival pathways might be below a critical threshold which eventually promotes neurodegeneration. We can only speculate about the reasons why in humans already single point mutations in parkin lead to a PD phenotype but mice seem to be able to tolerate a complete loss of parkin function. One reason might be that mice never reach the age when humans start to show PD symptoms; another reason might be more redundant compensatory pathways in the protein network in mice compared with humans. RET receptor loss in mice might mimic accelerated aging or increased stress by destabilizing the protective protein network in DA neurons.

Interestingly, in the RET-deficient background, DJ-1 seems to be essential only for DA cell body survival (6). Here, we found that parkin is important for cell body and axonal maintenance and has also a minor effect on dopamine levels in the striatum (Figures 1 and 2). Intact mitochondria are essential for the neuronal cell body and the axon to provide enough energy for their proper functioning. Therefore, a possible explanation for the difference between parkin and DJ-1 on fiber maintenance might be that, although both parkin and DJ-1 have been shown to protect mitochondria from toxic insults and ensure normal mitochondrial morphology and membrane potentiation, although this is through parallel signaling pathways (7), only parkin controls kinesin-dependent anterograde and dynein-dependent retrograde movements of mitochondria (40). This mitochondrial movement might be important to ensure a healthy pool of mitochondria in the axons essential for maintenance. Under physiological conditions, the mitochondrial transport in parkin-deficient mice seems to be unaffected, but under conditions of mitochondrial damage, the parkin-controlled mitochondrial transport mechanism becomes essential (40). Mitochondrial alterations in RET/parkin DKO mice might trigger the switch to the parkin-dependent mitochondrial transport, and parkin deficiency might thereby enhance axonal degradation. Further experiments are required to support this hypothesis.

The enhanced DA cell and fiber loss and mitochondrial dysfunction in the RET/parkin DKO mice most likely has a wide spread effect on mouse brain physiology and behavior. The reduced amount of energy and DA terminals can explain the lower level of dopamine in the striatum (Figure 2F) and dopamine release and reuptake. High ATP is required in DA neurons, for example, for the vesicular monoamine transporter, VMAT2, responsible for the dopamine uptake into presynaptic vesicles, to decrease the toxic oxidation-prone cytosolic dopamine and for removing the toxic amounts of calcium from the cytosol to the endoplasmic reticulum by high-affinity ATP-dependent transporters. Calcium gets into the cell by the sustained pacemaking activity of the L-type Ca 1.3 calcium channels in adult mice $(30,41)$. Therefore, reduced ATP levels might alter the total DA network, which controls many behaviors, such as movement, memory, motivation, and emotions $(22,42)$. Consistent with this rationale, we could detect changes in motor and emotional behavior in RET KO and RET/parkin DKO mice (Figure 3), which we did not observe in previously reported RET and RET/DJ-1 KO mice $(4,6)$.

The lack of GDNF to stimulate mitochondrial biogenesis is in contrast to other trophic and neurotrophic factors, such as nerve growth factor (NGF), brain-derived neurotrophic factor, and EGF, for which this has been reported (43). In addition, in dorsal root ganglia neurons, NGF was found to enhance neurite outgrowth and also to trigger local accumulation of mitochondria at sites in which NGF stimulates PI3K by binding to its high-affinity receptor, the receptor tyrosine kinase TRKA (43). Since RET can also stimulate axon outgrowth and guidance in motoneurons (18), it is exciting to investigate whether GDNF/RET signaling can also recruit mitochondria to specific locations and whether this determines the axon outgrowth direction and speed relevant for the regeneration after spinal cord injury $(44,45)$. Further research is required to address in detail how neurotrophic signaling receptors, such as RET but also parkin, regulate mitochondrial physiology, morphology, and transport, especially under mild stress conditions. One candidate gene regulated under these conditions might be OPA1, which has been identified as a NF- $\kappa \mathrm{B}$ target gene to promote mitochondrial fusion and to maintain mitochondrial integrity in cell culture (38) but seems not altered in the mouse models described here, at least in young mice under basal nonstress conditions (Supplemental Figure 3A). In the future, bioinformatics might allow for suggesting new NF- $\mathrm{BB}$ target genes by predicting DNA-binding sites in the genome, which can be tested concerning their effect on mitochondrial integrity in the different cellular contexts.

The presented data raise the question as to whether RET also crosstalks to other genes found to be mutated in familiar forms of PD. What can we expect, for example, from RET/PINK1 DKO mice? Since PINK1 is activating the E3 ligase activity of parkin (46) and is considered upstream of parkin, RET/PINK1 double-deficient mice might have a similar phenotype as that reported here for the RET-parkin DKO mice. Based on recent data that show that GDNF/ RET signaling can rescue muscle degeneration in PINK1-deficient Drosophila and mitochondrial defects in PINK1 knockdown human cells without inducing mitophagy or parkin recruitment (35), RET/ PINK1 DKO mice are worth generating and phenotyping.

Taken together, our data suggest that the RET signaling pathway is an essential part of the protein network altered in patients with PD and functionally connected to parkin (this study), PINK1 (35), DJ-1 (6), and $\alpha$-synuclein, which has been recently found to negatively regulate RET expression in PD (27). Changes in mitochondrial integrity seem to be a general problem leading to DA system degeneration. Since RET itself is required for mitochondrial integrity, GDNF/RET is one of the possible targets of the multiple-hit hypothesis for the development of PD and suggests that activating GDNF/RET signaling should be beneficial not only in the sporadic cases of PD but also in the genetic forms of PD, especially for patients with mutations in parkin, PINK1, and DJ-1. Although different clinical studies treating patients with PD with the RET ligand GDNF or neurturin have not yet convincingly shown a beneficial effect of this treatment - they just confirmed the lack of severe side effects by this approach - it is generally believed that this is 
at least in part due to technical problems of RET ligand application and the general study design that should be overcome in the near future $(47,48)$. Currently, a clinical phase I gene therapy trial is testing adeno-associated virus-encoded GDNF for the delivery of GDNF into the putamen of patients with PD (uniQure). This trial hopefully supports the safety of GDNF and the viral delivery approach, although the selection of patients with advanced PD with a strongly diminished number of GDNF receptor-expressing DA neurons might inhibit results showing the full beneficial potential of GDNF. The data presented here support the view that an early enhancement of GDNF/RET signaling and, especially, the

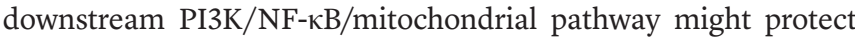
the residual DA neurons in patients with $\mathrm{PD}$ and perhaps even DA neurons transplanted as cell-replacement therapy.

Recently, it was described that GDNF/RET can activate the $\mathrm{NF}-\kappa \mathrm{B}$ pathway in neuroblastoma cells through binding of the adaptor protein SHC on the phosphorylated RET tyrosine 1062, which recruits GAB1 and activates PI3K and AKT (49). In addition, for the well-defined CREB activation by GDNF/RET, SHC is recruited to the phosphorylated RET tyrosine 1062 and binds Grb2 and SOS (49). RET tyrosine 1062 signaling through the $\mathrm{NF}-\kappa \mathrm{B}$ pathway is also considered essential to ensure the development and maintenance of the enteric nervous system and the inner ear neurons in mammals; therefore, GDNF/RET activation of the NF- $\mathrm{kB}$ pathway might not only be important for DA system maintenance to prevent PD but might also be essential to prevent Hirschsprung disease (megacolon disease) and congenital hearing loss in which GDNF/RET signaling is lost (50). Since all cells depend on mitochondrial respiration for energy production, the PI3K/NF- $\mathrm{B} /$ mitochondrial pathway described here to be downstream of GDNF/RET signaling might be reduced in Hirschsprung disease, congenital hearing loss, and PD and also in other neurodegenerative diseases in which alterations in mitochondrial function have been reported (7). In addition, this PI3K/ $\mathrm{NF}-\kappa \mathrm{B} /$ mitochondrial pathway downstream of GDNF/RET signaling might be hyperactive in multiple endocrine neoplasia type 2 cancer caused by RET gain-of-function mutations, such as the MEN2A and MEN2B mutations (51). Further experiments are needed to support this hypothesis.

Therefore, the converging parkin and GDNF/RET/PI3K/ $\mathrm{NF}-\kappa \mathrm{B} /$ mitochondrial pathway seems an interesting new target for developing more efficient drugs to treat diseases caused by or leading to RET receptor dysfunction.

\section{Methods}

Transgenic animals. All mice were maintained under standard animal housing conditions, with a 12-hour-day/night cycle and free access to food and water. The generation of RET floxed (Ret $t^{f / f}$ ) (18), Dat-Cre BAC (DCB-Cre) (19), parkin KO (16), and TH-GFP mice (52) was described previously, and mice were maintained on a C57BL/6J background. Details about the generation of transgenic human parkin-overexpressing mice can be found in the Supplemental Methods. Both male and female mice were used for different experiments at 3 to 6 months (adult) or 12 months or 24 months of age; whereas, for behavioral experiments only male mice were used at 12 or 24 months of age. The behavioral experiments are described in the Supplemental Methods.
Histology and immunohistochemistry. Tissue preparation, staining of DA neurons in the SNpc and VTA and of the DA innervations in the striatum, and their quantification by stereology (StereoInvestigator, MicroBrightField) and by an automatic fiber counting macro (Metamorph, Molecular Devices) were done by investigators who were blinded to the genotype of the sample as previously described (4) Primary antibodies used were a novel monoclonal rabbit anti-RET we made together with Epitomics (1:250), monoclonal mouse anti-TH (1:1,000; DiaSorin, 22941), monoclonal rat anti-DAT (1:500; Chemicon/Millipore, MAB369), and polyclonal goat anti-parkin (1:500; AbD Serotec, AHP495) (see also Supplemental Table 1).

Parkin and TH costained cells were analyzed using the cell counter plugin in the Image J program (NIH).

For soma area measurements, blinded investigators analyzed 100-150 cells per animal in the SNpc region from TH immune-stained coronal sections using a bright-field microscope with a $\times 63$ objective as described previously (6).

Tissue preparation for biochemistry. Unfixed brains were removed from mouse skulls, quickly frozen on dry ice, and stored at $-80^{\circ} \mathrm{C}$ until use. The frozen brains were cut into 1-mm-thick (for SNpc) and 2-mm-thick (for striatum) coronal sections ( $2 \mathrm{~mm}$ rostral or caudal to the interaural line), and $2 \mathrm{~mm}^{2}$ tissue circles were punched out with a sample corer (Fine Science Tools).

Tissue lyses and Western blot analysis. Tissue lyses was performed as described in the Supplemental Methods, and Western blot analysis on dissected SNpc and striatum samples and SH-SY5Y cells was done as described previously $(4,53)$. In order to detect specific antigens, blots were incubated with antibodies mentioned in Supplemental Table 2.

Total striatal dopamine measurements. HPLC analysis of total dopamine and its metabolites in the dissected striatal tissue was done as described previously (54), with minor modifications (described in the Supplemental Methods), by investigators who were blinded to the genotype of the samples

Electrophysiology. GFP-positive neurons in the SNpc of TH-GFP mice (52) crossed with the parkin and RET mutant mice as well as the patched cells injected with an Alexa Fluor 594-filled patch pipette (resistance 4-5 MOhm) were visualized by confocal microscopy. After the whole-cell configuration was established, capacitance was recorded in voltage clamp mode, while membrane voltage was recorded in current clamp mode using a patch-clamp amplifier and software (Multiclamp 700B and PClamp 10, Molecular Devices). Series resistance was compensated by about $50 \%$.

Mitochondrial enrichment and complex I activity measurement. Mitochondrial enrichment from mouse brain SNpc and siRNA-transfected SH-SY5Y cells (cultured for 24 hours in $3 \mathrm{mM}$ glucose medium before harvesting) was done as described previously (31), with minor modifications (described in the Supplemental Methods). Complex I enzyme activity assay was performed using the Complex I Enzyme Activity Microplate Assay Kit (ab109721, Abcam) according to the manufacturer's instructions, with $30 \mu \mathrm{g}$ protein for tissue and $50 \mu \mathrm{g}$ protein for SH-SY5Y cells from the enriched mitochondrial preparations. Data from one extra RET KO mouse were excluded as outliers, since its distance from the median exceeds 1.5 times the interquartile range in the box-and-whiskers plot in Prism (Figure 4I).

Measurement of cellular ATP levels in mouse brain tissue. Dissected SNpc tissue samples were homogenized in $200 \mu$ of precooled ATP assay buffer provided in the ATP Assay Kit (ab83355, Abcam) using a 
Teflon dounce. A small volume of the homogenate was used for protein concentration determination using BCA (Pierce), and the rest was centrifuged at $14,000 \mathrm{~g}$ for 15 minutes. $50 \mu \mathrm{l}$ of the supernatant was used to perform fluorometric ATP assay according to the manufacturer's instructions. The values obtained were normalized to total protein levels.

Measurement of cellular ATP levels in SH-SY5Y cells. Measurement of cellular ATP levels was carried out as described before using the ATP Bioluminescence Assay Kit HS II (Roche Applied Science) according to the manufacturer's instructions (39). Briefly, SH-SY5Y cells were reverse transfected with the siRNAs indicated below. 24 hours before harvesting, the culture medium was replaced by medium containing $3 \mathrm{mM}$ glucose. Cells were washed twice with PBS, scraped off the plate, and lysed according to the manufacturer's instructions. Bioluminescence of the samples was determined using an LB96V luminometer (Berthold Technologies), analyzed with WinGlow Software (Berthold Technologies), and normalized to total protein levels.

Plasmids. The following constructs were described previously: human parkin (39); human RET wild-type, KiD, and MEN2A (55); FLAG-IK $\beta \Delta \mathrm{N}$ (56); IKK $\beta$ (57); plasmid encoding mitochondrially targeted DsRed (Mito-DsRed) (58); and PI3Kwt and PI3Kmyr (Invitrogen).

Cell cultures, treatments, and RNA interference. SH-SY5Y (DSMZ no. ACC 209) cells were cultivated in DMEM (Lonza) supplemented with $15 \%$ FCS (Sigma-Aldrich) and maintained at $37^{\circ} \mathrm{C}, 5 \% \mathrm{CO}_{2}$. For RNA interference, cells were reverse transfected with stealth siRNA oligos, human RET (HSS109181, Invitrogen) and human parkin (HSS107594, Invitrogen), using Lipofectamine RNAiMAX (Invitrogen). Cells were harvested at day 3 after transfection. When coexpression of other constructs was required in the presence of acute knockdown, Mito-DsRed was used at a concentration of $0.2 \mu \mathrm{g}$ as a cotransfection control. For experiments involving acute stimulation of endogenous RET, cells were stimulated for 3 to 4 hours with hGDNF (Shenandoah Biotechnology Inc.) and hGFR $\alpha 1$ (R\&D Systems) at a final concentration of $100 \mathrm{ng} / \mathrm{ml}$. Signaling pathway inhibitors, MEK1+2 inhibitor U0126 (Cell Signaling Technology), PI3K inhibitor LY294002 (Cell Signaling Technology), and MEK1 inhibitor PD98059 (Cayman Chemicals), were added 90 minutes prior to control or parkin siRNA treatment and GDNF/GFR $\alpha$ stimulation and were used at a final concentration of 20,10 , and $10 \mu \mathrm{M}$, respectively.

Analysis of mitochondrial morphology in cultured cells. Cells previously grown on 15-mm glass coverslips were fixed with 3.7\% PFA in PBS for 10 minutes, permeabilized with 0.1\% Triton X-100 PBS for 5 minutes, blocked with 5\% BSA in PBS for 10 minutes, and incubated with polyclonal rabbit anti-TOM20 antibody (1:500; Santa Cruz Biotechnology) in 5\% BSA in PBS overnight at $4^{\circ} \mathrm{C}$. After extensive washing, fixed cells were incubated with the corresponding Alexa Fluor-conjugated secondary antibodies for 2 hours at room temperature. Nuclei were counterstained with DAPI. Images were acquired with a Zeiss LSM710 confocal microscope with a $\times 63$ oil objective (NA 1.4). Classification of the mitochondrial morphology was performed according to the following criteria: cells displaying an intact tubular mitochondrial network were classified as tubular; cells with globular or rod-like mitochondria were classified as fragmented; and cells showing a cluster of curly and thicker mitochondria were classified as condensed. Quantifications were based on duplicates of at least 3 independent experiments.

Real-time RT-PCR. Knockdown efficiency of parkin and Ret was evaluated by real-time RT-PCR with the 7500 Fast Real-Time System (Applied Biosystems) as previously described (59) (see the Supplemental Methods for primer sequences).

Statistics. All quantitative data were analyzed using GraphPad Prism 5 (GraphPad Software) and are represented as mean \pm SEM. The significance of alterations was generally tested with ANOVA followed by Newman-Keuls post-hoc test. In a few cases, 2-tailed $t$ test was used, and this is mentioned in the figure legends. A $P$ value of smaller than 0.05 was considered significant.

Study approval. All animal (mouse) experiments were performed in accordance with the German and European Animal Welfare Act and with the approval of local authorities of the city-state Hamburg (Behörde für Gesundheit und Verbraucherschutz, Fachbereich Veterinärwesen) and the animal care committee of the University Medical Center Hamburg-Eppendorf as well as the state of Bavaria (Regierung von Oberbayern, Umwelt, Gesundheit und Verbraucherschutz, Fachbereich Veterinärmedizin).

\section{Acknowledgments}

We thank A. Brice, O. Corti, G. Schütz, F. Tronche, P. Soriano, and K. Kobayashi for mouse donation; M. Saarma, R. Klein, K. Duncan, and R. Stevens for helpful comments on the manuscript; and P. Putthoff, M. Wildung, J. Lüdemann, M. Schweizer, E. Szpotowicz, I. Hermans-Borgmeyer, S. Homann, A. Merseburg, F. Morellini, R. Fliegert, and the members of the mouse facility at the University Medical Center Hamburg-Eppendorf for excellent technical assistance. This study was partly supported by grants from the DFG (KR 3529/4-1 to E.R. Kramer, LO771/6 to C. Lohr, SFB 596 "Molecular Mechanisms of Neurodegeneration" to K.F. Winklhofer and J. Tatzelt), the town of Hamburg (Lexi to E.R. Kramer), the German Federal Ministry of Education and Research (BMBF) (National Genome Research Network [NGFN] plus "Functional Genomics of Parkinson's Disease" to K.F. Winklhofer), and the Helmholtz Alliance ("Mental Health in an Ageing Society" to K.F. Winklhofer).

Address correspondence to: Edgar R. Kramer, Research Group Development and Maintenance of the Nervous System, Center for Molecular Neurobiology Hamburg (ZMNH), University Medical Center Hamburg-Eppendorf (UKE), Falkenried 94, 20251 Hamburg, Germany. Phone: 49.0.40.7410.55354; E-mail: kramer@ zmnh.uni-hamburg.de.

Anne Kathrin Müller-Rischart's present address is: Center for Integrated Protein Science, Fakultät für Biologie, Ludwig-Maximilians-Universität München, Planegg-Martinsried, Germany.

Margit Miesbauer's present address is: Roche Pharma AG, Grenzach-Wyhlen, Germany.
1. Dawson TM, Ko HS, Dawson VL. Genetic animal models of Parkinson's disease. Neuron. 2010;66(5):646-661.

2. Martin I, Dawson VL, Dawson TM. Recent advances in the genetics of Parkinson's disease.
Annu Rev Genomics Hum Genet. 2011;12:301-325.

3. Sulzer D. Multiple hit hypotheses for dopamine neuron loss in Parkinson's disease. Trends Neurosci. 2007;30(5):244-250.

4. Kramer ER, et al. Absence of Ret signaling in mice causes progressive and late degeneration of the nigrostriatal system. PLoS Biol. 2007;5(3):e39.

5. Pascual A, Hidalgo-Figueroa M, Piruat JI, Pintado CO, Gomez-Diaz R, Lopez-Barneo J. 
Absolute requirement of GDNF for adult catecholaminergic neuron survival. Nat Neurosci. 2008;11(7):755-761.

6. Aron L, Klein P, Pham TT, Kramer ER, Wurst W, Klein R. Pro-survival role for Parkinson's associated gene DJ-1 revealed in trophically impaired dopaminergic neurons. PLoS Biol. 2010;8(4):e1000349.

7. Exner N, Lutz AK, Haass C, Winklhofer KF. Mitochondrial dysfunction in Parkinson's disease: molecular mechanisms and pathophysiological consequences. EMBO J. 2012;31(14):3038-3062.

8. Corti O, Brice A. Mitochondrial quality control turns out to be the principal suspect in parkin and PINK1-related autosomal recessive Parkinson's disease. Curr Opin Neurobiol. 2013;23(1):100-108.

9. Youle RJ, Narendra DP. Mechanisms of mitophagy. Nat Rev Mol Cell Biol. 2011;12(1):9-14.

10. Bian M, et al. Overexpression of parkin ameliorates dopaminergic neurodegeneration induced by 1-methyl-4-phenyl-1,2,3,6-tetrahydropyridine in mice. PLoS One. 2012;7(6):e39953.

11. Lo Bianco C, et al. Lentiviral vector delivery of parkin prevents dopaminergic degeneration in an $\alpha$-synuclein rat model of Parkinson's disease. Proc Natl Acad Sci U S A. 2004;101(50):17510-17515.

12. Yamada M, Mizuno Y, Mochizuki H. Parkin gene therapy for $\alpha$-synucleinopathy: a rat model of Parkinson's disease. Hum Gene Ther. 2005;16(2):262-270.

13. Fallon $\mathrm{L}$, et al. A regulated interaction with the UIM protein Eps15 implicates parkin in EGF receptor trafficking and $\mathrm{PI}(3) \mathrm{K}$-Akt signalling. Nat Cell Biol. 2006;8(8):834-842.

14. Fusco D, et al. The RET51/FKBP52 complex and its involvement in Parkinson disease. Hum Mol Genet. 2010;19(14):2804-2816.

15. Farrer MJ. Genetics of Parkinson disease: paradigm shifts and future prospects. Nat Rev Genet. 2006;7(4):306-318.

16. Itier JM, et al. Parkin gene inactivation alters behaviour and dopamine neurotransmission in the mouse. Hum Mol Genet. 2003;12(18):2277-2291.

17. Kowsky S, et al. RET signaling does not modulate MPTP toxicity but is required for regeneration of dopaminergic axon terminals. Proc Natl Acad Sci US A. 2007;104(50):20049-20054.

18. Kramer ER, et al. Cooperation between GDNF/ Ret and ephrinA/EphA4 signals for motoraxon pathway selection in the limb. Neuron. 2006;50(1):35-47.

19. Parlato R, Rieker C, Turiault M, Tronche F, Schutz G. Survival of DA neurons is independent of CREM upregulation in absence of CREB. Genesis. 2006;44(10):454-464.

20. Turiault M, et al. Analysis of dopamine transporter gene expression pattern - generation of DAT-iCre transgenic mice. FEBS J. 2007;274(14):3568-3577.

21. Goldberg MS, et al. Parkin-deficient mice exhibit nigrostriatal deficits but not loss of dopaminergic neurons. J Biol Chem. 2003;278(44):43628-43635.

22. Bjorklund A, Dunnett SB. Dopamine neuron systems in the brain: an update. Trends Neurosci. 2007;30(5):194-202.

23. Fearnley JM, Lees AJ. Ageing and Parkinson's dis- ease: substantia nigra regional selectivity. Brain. 1991;114(5):2283-2301.

24. Dunkley PR, Bobrovskaya L, Graham ME, von Nagy-Felsobuki EI, Dickson PW. Tyrosine hydroxylase phosphorylation: regulation and consequences. J Neurochem. 2004;91(5):1025-1043.

25. Sagna A, Gallo JJ, Pontone GM. Systematic review of factors associated with depression and anxiety disorders among older adults with Parkinson's disease. Parkinsonism Relat Disord. 2014;20(7):708-715.

26. Beitz JM. Parkinson's disease: a review. Front Biosci (Schol Ed). 2014;6:65-74.

27. Decressac M, Kadkhodaei B, Mattsson B, Laguna A, Perlmann T, Bjorklund A. $\alpha$-Synuclein-induced down-regulation of Nurr1 disrupts GDNF signaling in nigral dopamine neurons. Sci Transl Med. 2012;4(163):163ra56.

28. Greenbaum L, et al. Perspective: Identification of genetic variants associated with dopaminergic compensatory mechanisms in early Parkinson's disease. Front Neurosci. 2013;7(52):1-9.

29. Gautier CA, Kitada T, Shen J. Loss of PINK1 causes mitochondrial functional defects and increased sensitivity to oxidative stress. Proc Nat Acad Sci U S A. 2008;105(32):11364-11369.

30. Gispert S, et al. Parkinson phenotype in aged PINK1-deficient mice is accompanied by progressive mitochondrial dysfunction in absence of neurodegeneration. PLoS One. 2009;4(6):e5777.

31. Palacino JJ, et al. Mitochondrial dysfunction and oxidative damage in parkin-deficient mice. J Biol Chem. 2004;279(18):18614-18622.

32. Stichel CC, Zhu XR, Bader V, Linnartz B, Schmidt $S$, Lubbert H. Mono- and double-mutant mouse models of Parkinson's disease display severe mitochondrial damage. Hum Mol Genet. 2007;16(20):2377-2393.

33. Pilsl A, Winklhofer KF. Parkin, PINK1 and mitochondrial integrity: emerging concepts of mitochondrial dysfunction in Parkinson's disease. Acta Neuropathol. 2012;123(2):173-188.

34. Irrcher I, et al. Loss of the Parkinson's disease-linked gene DJ-1 perturbs mitochondrial dynamics. Hum Mol Genet. 2010;19(19):3734-3746.

35. Klein P, et al. Ret rescues mitochondrial morphology and muscle degeneration of Drosophila Pink1 mutants. EMBO J. 2014;33(4):341-355.

36. Deas E, Wood NW, Plun-Favreau H. Mitophagy and Parkinson's disease: the PINK1-parkin link. Biochim Biophys Acta. 2011;1813(4):623-633.

37. Henn IH, et al. Parkin mediates neuroprotection through activation of IкB kinase/ nuclear factor-kappaB signaling. J Neurosci. 2007;27(8):1868-1878.

38. Muller-Rischart AK, et al. The E3 ligase parkin maintains mitochondrial integrity by increasing linear ubiquitination of NEMO. Mol Cell. 2013;49(5):908-921.

39. Lutz AK, et al. Loss of parkin or PINK1 function increases Drp1-dependent mitochondrial fragmentation.J Biol Chem. 2009; 284(34):22938-22951.

40. Schwarz TL. Mitochondrial trafficking in neurons. Cold Spring Harb Perspect Biol. 2013;5(6):a011304.
41. Surmeier DJ, Schumacker PT. Calcium, bioenergetics, and neuronal vulnerability in Parkinson's disease. J Biol Chem. 2013;288(15):10736-10741.

42. Leknes S, Tracey I. A common neurobiology for pain and pleasure. Nat Rev Neurosci. 2008;9(4):314-320.

43. Cheng A, Hou Y, Mattson MP. Mitochondria and neuroplasticity. ASN Neuro. 2010;2(5):e00045.

44. Mills CD, Allchorne AJ, Griffin RS, Woolf CJ, Costigan M. GDNF selectively promotes regeneration of injury-primed sensory neurons in the lesioned spinal cord. Mol Cell Neurosci. 2007;36(2):185-194.

45. Zhang L, et al. GDNF-enhanced axonal regeneration and myelination following spinal cord injury is mediated by primary effects on neurons. Glia. 2009;57(11):1178-1191.

46. Koyano F, et al. Ubiquitin is phosphorylated by PINK1 to activate parkin. Nature. 2014;510(7503):162-166

47. Meissner WG, et al. Priorities in Parkinson's disease research. Nat Rev Drug Discov. 2011;10(5):377-393.

48. Kordower JH, Bjorklund A. Trophic factor gene therapy for Parkinson's disease. Mov Disord. 2013;28(1):96-109.

49. Hayashi H, et al. Characterization of intracellular signals via tyrosine 1062 in RET activated by glial cell line-derived neurotrophic factor. Oncogene. 2000;19(39):4469-4475.

50. Ohgami N, et al. c-Ret-mediated hearing losses. Int JClin Exp Pathol. 2012;5(1):23-28.

51. Santoro M, et al. Activation of RET as a dominant transforming gene by germline mutations of MEN2A and MEN2B. Science. 1995;267(5196):381-383.

52. Sawamoto K, et al. Visualization, direct isolation, and transplantation of midbrain dopaminergic neurons. Proc Natl Acad Sci U S A. 2001;98(11):6423-6428.

53. Winklhofer KF, Henn IH, Kay-Jackson PC, Heller U, Tatzelt J. Inactivation of parkin by oxidative stress and C-terminal truncations: a protective role of molecular chaperones. J Biol Chem. 2003;278(47):47199-47208.

54. Yang L, Beal MF. Determination of neurotransmitter levels in models of Parkinson's disease by HPLC-ECD. Methods Mol Biol. 2011;793:401-415.

55. Rossel M, et al. Distinct biological properties of two RET isoforms activated by MEN 2A and MEN 2B mutations. Oncogene. 1997;14(3):265-275.

56. Krappmann D, Wulczyn FG, Scheidereit C. Different mechanisms control signal-induced degradation and basal turnover of the NF-kB inhibitor IкBo in vivo. EMBO J. 1996;15(23):6716-6726.

57. Zandi E, Rothwarf DM, Delhase M, Hayakawa M, Karin M. The IкB kinase complex (IKK) contains two kinase subunits, IKK $\alpha$ and IKK $\beta$, necessary for

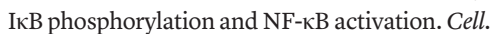
1997;91(2):243-252.

58. Okita C, Sato M, Schroeder T. Generation of optimized yellow and red fluorescent proteins with distinct subcellular localization. Biotechniques. 2004;36(3):418-424

59. Bouman L, et al. Parkin is transcriptionally regulated by ATF 4: evidence for an interconnection between mitochondrial stress and ER stress. Cell Death Differ. 2011;18(5):769-782. 